\title{
PORTRAITS OF FRAMES
}

\author{
AKRAM ALDROUBI
}

(Communicated by Palle E. T. Jorgensen)

\begin{abstract}
We introduce two methods for generating frames of a Hilbert space $\mathscr{H}$. The first method uses bounded operators on $\mathscr{H}$. The other method uses bounded linear operators on $l_{2}$ to generate frames of $\mathscr{H}$. We characterize all the mappings that transform frames into other frames. We also show how to construct all frames of a given Hilbert space $\mathscr{H}$, starting from any given one. We illustrate the results by giving some examples from multiresolution and wavelet theory.
\end{abstract}

\section{INTRODUCTION}

A frame of a Hilbert space $\mathscr{H}$ is a sequence $\left\{\phi_{n}\right\}_{n \in Z}$ of vectors such that for any vector $v \in \mathscr{H}$

$$
A\|v\|_{\mathscr{Z}}^{2} \leq \sum_{n \in Z}\left|\left\langle v, \phi_{n}\right\rangle_{\mathscr{H}}\right|^{2} \leq B\|v\|_{\mathscr{H}}^{2},
$$

where the frame bounds $A$ and $B$ are positive constants. This notion has been introduced by R. J. Duffin and A. C. Schaeffer in the context of nonharmonic Fourier series [10]. It is the generalization of the concept of orthnormal sets in Hilbert spaces. Specifically, if $\left\{\phi_{n}\right\}_{n \in Z}$ is a frame of $\mathscr{H}$, then any vector $v \in \mathscr{H}$ can be represented as [10]

$$
v=\sum_{n \in Z}\left\langle S^{-1} v, \phi_{n}\right\rangle_{\mathscr{C}} \phi_{n},
$$

where $S^{-1}$ is the inverse of the selfadjoint operator $S$ on $\mathscr{H}$, defined by

$$
S u:=\sum_{n \in Z}\left\langle u, \phi_{n}\right\rangle_{\mathscr{L}} \phi_{n} .
$$

Identity (2) can also be written in terms of the dual frame $\left\{\stackrel{\circ}{\phi}_{n}=S^{-1} \phi_{n}\right\}_{n \in Z}$ [10]:

$$
v=\sum_{n \in Z}\left\langle v, \stackrel{\circ}{\phi}_{n}\right\rangle_{\mathscr{K}} \phi_{n} .
$$

Received by the editors October 1, 1993; presented to the SPIE meeting, July 15, 1993.

1991 Mathematics Subject Classification. Primary 42C99, 46C99.

Key words and phrases. Frames, frame-preserving mappings, affine frames, multiresolution, wavelets. 
Thus, any unconditional basis of $\mathscr{H}$ is also a frame of $\mathscr{H}$, but the converse is not true [5, 9].

In this paper, we characterize the frame-preserving mappings that transform frames of $\mathscr{H}$ into other frames of $\mathscr{H}$. In $\S 2$, we give the necessary and sufficient conditions satisfied by the frame-preserving operators on $\mathscr{H}$. We also characterize the operators that generate frames of $\mathscr{H}_{1} \subset \mathscr{H}$. In $\S 3$, we describe the class of operators on $l_{2}$ that generates all the frames of a Hilbert space $\mathscr{H}$. These operators on $l_{2}$ relate any two frames of $\mathscr{H}$. The results can be used to obtain a general method for constructing frames with prescribed properties. In particular, we apply the results to affine frames and give some examples from multiresolution and wavelet theory [12].

\section{MAPPING ON $\mathscr{H}$ FOR THE CONSTRUCTION OF FRAMES}

2.1. Construction of frames of $\mathscr{H}$. Starting from a frame $\left\{\phi_{n}\right\}_{n \in Z} \subset \mathscr{H}$, we wish to find methods to obtain other frames of $\mathscr{H}$. Such methods would allow the construction of frames with some prescribed properties that are suited for specific applications. One approach is to construct a sequence $\theta_{n}=T \phi_{n}$, where $T$ is a bounded linear operator on $\mathscr{H}$. If the range of $T$ is not dense in $\mathscr{H}$, then we can find a nonzero vector $w$ which is orthogonal to the range of $T$ : $w \in C \operatorname{los}(\operatorname{Ran}(T))^{\perp}$. Clearly, $w$ cannot be a linear combination of $\left\{\theta_{n}\right\}_{n \in Z}$. Thus, for $\left\{\theta_{n}\right\}_{n \in Z}$ to be a frame, it is necessary for the range of $T$ to be dense in $\mathscr{H}$. However, this condition is not sufficient. A necessary and sufficient condition for $\left\{\theta_{n}=T \phi_{n}\right\}_{n \in Z}$ to be a frame is that the adjoint operator $T^{*}$ be coercive, as in the following theorem.

Theorem 1. Let $\left\{\phi_{n}\right\}_{n \in Z}$ be a frame of $\mathscr{H}$ with frame bounds $0<A \leq B$. If $T$ is a bounded linear operator from $\mathscr{H}$ into $\mathscr{H}$, then $\left\{\theta_{n}=T \phi_{n}\right\}_{n \in Z}$ is a frame for $\mathscr{H}$ if and only if there exists a positive constant $\gamma$ such that the adjoint operator $T^{*}$ satisfies

$$
\left\|T^{*} v\right\|_{\mathscr{H}}^{2} \geq \gamma\|v\|_{\mathscr{H}}^{2}
$$

Remark. Condition (5) implies that $T^{*}$ is injective; i.e., the null space of $T^{*}$ consists of the zero vector: $\operatorname{null}\left(T^{*}\right)=\{0\}$. Thus, the range of $T$ is dense in $\mathscr{H}$. It should also be noted that positive selfadjoint operators always take frames of $\mathscr{H}$ into other frames.

Proof. Sufficiency. We use (1) with the frame bound $A$ and the property of the adjoint to obtain that

$$
\gamma A\|v\|_{\mathscr{H}}^{2} \leq A\left\|T^{*} v\right\|_{\mathscr{H}}^{2} \leq \sum_{n \in Z}\left|\left\langle v, T \phi_{n}\right\rangle_{\mathscr{H}}\right|^{2}=\sum_{n \in Z}\left|\left\langle T^{*} v, \phi_{n}\right\rangle_{\mathscr{H}}\right|^{2} .
$$

A similar estimate with the frame bound $B$ yields

$$
\sum_{n \in Z}\left|\left\langle v, T \phi_{n}\right\rangle_{\mathscr{H}}\right|^{2}=\sum_{n \in Z}\left|\left\langle T^{*} v, \phi_{n}\right\rangle_{\mathscr{H}}\right|^{2} \leq B\left\|T^{*} v\right\|_{\mathscr{H}}^{2} \leq B\left\|T^{*}\right\|^{2}\|v\|_{\mathscr{H}}^{2},
$$

which completes the "if" part of the proof.

Necessity. We assume that $\left\{\theta_{n}=T \phi_{n}\right\}_{n \in Z}$ is a frame of $\mathscr{H}$ with frame bounds $0<a \leq b$ so that

$$
a\|v\|_{\mathscr{P}}^{2} \leq \sum_{n \in Z}\left|\left\langle v, T \phi_{n}\right\rangle_{\mathscr{L}}\right|^{2} \leq b\|v\|_{\mathscr{P}}^{2}
$$


Using the upper frame bound $B$ of $\left\{\phi_{n}\right\}_{n \in Z}$ and the left inequality of (6), we obtain

$$
a\|v\|_{\mathscr{H}}^{2} \leq \sum_{n \in Z}\left|\left\langle v, T \phi_{n}\right\rangle_{\mathscr{H}}\right|^{2}=\sum_{n \in Z}\left|\left\langle T^{*} v, \phi_{n}\right\rangle_{\mathscr{C}}\right|^{2} \leq B\left\|T^{*} v\right\|_{\mathscr{H}}^{2},
$$

from which (5) follows with $\gamma=a / B$.

2.2. Construction of frames on subspaces $\mathscr{H}_{1} \subset \mathscr{H}$. Using continuous linear operators on $\mathscr{H}$, a construction similar to the previous one can be used to create frames of subspaces $\mathscr{H}_{1} \subset \mathscr{H}$. We have the following result:

Theorem 2. Let $\mathscr{H}_{1} \subset \mathscr{H}$ be a closed subspace, and let $\left\{\phi_{n}\right\}_{n \in Z}$ be a frame of $\mathscr{H}$. If $T$ is a bounded linear operator from $\mathscr{H}$ into $\mathscr{H}_{1}$, then $\left\{\theta_{n}=T \phi_{n}\right\}_{n \in Z}$ is a frame of $\mathscr{H}_{1}$ if and only if the restriction $T_{r}^{*}$, of $T^{*}$ to $\mathscr{H}_{1}$, is coercive, i.e., there exists a positive constant $\gamma$ such that

$$
\left\|T^{*} v\right\|_{\mathscr{H}}^{2} \geq \gamma\|v\|_{\mathscr{H}}^{2}, \quad \forall v \in \mathscr{H}_{1} .
$$

If the operator $T=P$ in Theorem 2 is an orthogonal projection from $\mathscr{H}$ into $\mathscr{H}_{1}$, then $P^{*}=P$ and $\|P v\|_{\mathscr{H}}^{2}=\|v\|_{\mathscr{H}}^{2}$ for all $v \in \mathscr{H}_{1}$. Thus, from the previous theorem, we immediately get that $\left\{\theta_{n}=P \phi_{n}\right\}_{n \in Z}$ is a frame of $\mathscr{H}_{1}$. To obtain a dual frame, we simply write $v \in \mathscr{H}_{1}$ in terms of the frame $\left\{\phi_{n}\right\}_{n \in Z}$ of $\mathscr{H}$ and use the properties of the projection operator $P$ to get

$$
v=P v=\sum_{n \in Z}\left\langle v, \stackrel{\circ}{\phi}_{n}\right\rangle_{\mathscr{H}} \phi_{n}=\sum_{n \in Z}\left\langle P v, \stackrel{\circ}{\phi}_{n}\right\rangle_{\mathscr{H}} \phi_{n}=\sum_{n \in Z}\left\langle v, P \stackrel{\circ}{\phi_{n}}\right\rangle_{\mathscr{H}} P \phi_{n} .
$$

Thus, $\left\{\stackrel{\circ}{\theta}_{n}=P \stackrel{\circ}{\theta}_{n}\right\}_{n \in Z}$ and $\left\{\theta_{n}=P \phi_{n}\right\}_{n \in Z}$ are dual frames in $\mathscr{H}_{1}$, and any vector $v \in \mathscr{H}_{1}$ can be written in terms of this pair of dual frames as

$$
v=\sum_{n \in Z}\left\langle v, \stackrel{\circ}{\theta}_{n}\right\rangle_{\mathscr{H}} \theta_{n}=\sum_{n \in Z}\left\langle v, \theta_{n}\right\rangle_{\mathscr{H}} \stackrel{\circ}{\theta}_{n}
$$

Thus we have proven:

Theorem 3. If $\mathscr{H}_{1} \subset \mathscr{H}$ is a closed subspace of $\mathscr{H}$ and if $P$ is the orthogonal projection on $\mathscr{H}_{1}$, then $\left\{\theta_{n}=P \phi_{n}\right\}_{n \in Z}$ and $\left\{\stackrel{\circ}{\theta}_{n}=P \stackrel{\circ}{\phi}_{n}\right\}_{n \in Z}$ are dual frames of $\mathscr{H}_{1}$. Moreover, the frame bounds $A$ and $B$ of $\left\{\phi_{n}\right\}_{n \in Z}$ are also frame bounds for $\left\{\theta_{n}\right\}_{n \in Z}$.

Thus, starting from an orthogonal basis of $\mathscr{H}$, we can construct frames of $\mathscr{H}_{1}$ that are tight (i.e., $A=B$ ). This fact enables the construction of shiftinvariant tight frames. It also allows the construction of frame multiresolution approximations (FMRA) [7] that are tight (cf. $\S 4.1$ ).

\section{MAPPINGS ON $l_{2}$ FOR THE CONSTRUCTION OF FRAMES OF $\mathscr{H}$}

Let $U$ be a bounded linear operator on $l_{2}$, and let the sequence $\Phi=$ $\left(\ldots, \phi_{n-1}, \phi_{n}, \phi_{n+1}, \ldots\right)$ be a frame of $\mathscr{H}$. We can use $U$ to construct the sequence $\boldsymbol{\Theta}=U \boldsymbol{\Phi}$, defined by $\boldsymbol{\Theta}=\left(\ldots, \theta_{n-1}, \theta_{n}, \theta_{n+1}, \ldots\right)$ and

$$
\theta_{n}=\sum_{j \in Z} u_{n j} \phi_{j}
$$


where $u_{i j}=\left\langle U e_{j}, e_{i}\right\rangle_{l_{2}}$ and $e_{i} \in l_{2}$ is the canonical basis vector (i.e., $e_{i}(i)=1$, $\left.e_{i}(j)=0, i \neq j\right)$. Since $\left\{\phi_{n}\right\}_{n \in Z}$ is a frame of $\mathscr{H}$ with bounds $A$ and $B$, and since $U$ is a bounded operator, the vectors $\theta_{n}$ are well defined, and we have

$$
\left\|\theta_{n}\right\|_{\mathscr{X}}^{2} \leq B\|U\|^{2}
$$

In general, $\left\{\theta_{n}\right\}_{n \in Z}$ does not constitute a frame of $\mathscr{H}$ (e.g., $U=0$ ). However, under appropriate conditions on $U$, the sequence $\boldsymbol{\theta}=\left(\ldots, \theta_{n-1}, \theta_{n}, \theta_{n+1}, \ldots\right)$ is a frame of $\mathscr{H}$. In order to characterize the operators that transform frames of $\mathscr{H}$ into other frames of $\mathscr{H}$, we introduce the set of linear operators $p(\cdot, \Lambda)$ from $\mathscr{H}$, taking values in $l_{2}$, defined by

$$
p(v, \Lambda)=\left(\ldots,\left\langle v, \lambda_{n-1}\right\rangle_{\mathscr{H}},\left\langle v, \lambda_{n}\right\rangle_{\mathscr{H}},\left\langle v, \lambda_{n+1}\right\rangle_{\mathscr{H}}, \ldots\right), \quad \forall v \in \mathscr{H},
$$

where $\Lambda=\left(\ldots, \lambda_{n-1}, \lambda_{n}, \lambda_{n+1}, \ldots\right)$ is any fixed sequence that forms a frame of $\mathscr{H}$. The operator $p$ is a bounded linear operator from $\mathscr{H}$ into $l_{2}$, since

$$
\|p(v, \Lambda)\|_{l_{2}}^{2}=\sum_{n \in Z}\left|\left\langle v, \lambda_{n}\right\rangle_{\mathscr{P}}\right|^{2} \leq B_{\Lambda}\|v\|_{\mathscr{K}}^{2},
$$

where $B_{\Lambda}$ is an upper frame bound for $\left\{\lambda_{n}\right\}_{n \in Z}$. Now, the above derivations allow us to characterize all the operators $U$ on $l_{2}$ that transform, by procedure (10), frames of $\mathscr{H}$ into other frames of $\mathscr{H}$ :

Theorem 4. Let $\left\{\phi_{n}\right\}_{n \in Z}$ be a frame of $\mathscr{H}$, and $U$ be a bounded linear operator from $l_{2}$ into itself. Then $\left\{\theta_{n}=(U \Phi)_{n}\right\}_{n \in Z}$ is a frame of $\mathscr{H}$ if and only if there exists a constant $\gamma>0$ such that for all $x \in X=\operatorname{Range}(p(\cdot, \Phi))$ we have

$$
\|U x\|_{l_{2}}^{2} \geq \gamma\|x\|_{l_{2}}^{2}, \quad \forall x \in X .
$$

Proof. Sufficiency. From (1) and (11) we get

$$
A\|v\|_{\mathscr{P}}^{2} \leq\|p(v, \Phi)\|_{l_{2}}^{2} \leq B\|v\|_{\mathscr{P}}^{2} \text {. }
$$

A simple computation yields

$$
p(v, \boldsymbol{\Theta})=U p(v, \Phi)
$$

from which we obtain the two inequalities

$$
\begin{gathered}
A \gamma\|v\|_{\mathscr{X}}^{2} \leq \gamma\|p(v, \Phi)\|_{l_{2}}^{2} \leq\|U p(v, \Phi)\|_{l_{2}}^{2}=\|p(v, \boldsymbol{\theta})\|_{l_{2}}^{2}, \\
\|p(v, \boldsymbol{\theta})\|_{l_{2}}^{2}=\|U p(v, \Phi)\|_{l_{2}}^{2} \leq\|U\|\|p(v, \Phi)\|_{l_{2}}^{2} \leq B\|U\|\|v\|_{\mathscr{K}}^{2} .
\end{gathered}
$$

Thus $\left\{\theta_{n}\right\}_{n \in Z}$ is a frame for $\mathscr{H}$ with frame constants $a=A \gamma$ and $b=\|U\| B$.

Necessity. For the converse, we assume that $\left\{\theta_{n}\right\}_{n \in Z}$ is a frame. Thus, using (15), we have

$$
a\|v\|_{\mathscr{Z}}^{2} \leq\|p(v, \boldsymbol{\theta})\|_{l_{2}}^{2}=\|U p(v, \boldsymbol{\Phi})\|_{l_{2}}^{2} \leq b\|v\|_{\mathscr{K}}^{2},
$$

where $a>0$ and $b>0$ are frame bounds of $\left\{\theta_{n}\right\}_{n \in Z}$. We then use the left inequality of (18) and the right inequality of (14) to obtain

$$
\frac{a}{B}\|p(v, \Phi)\|_{l_{2}}^{2} \leq\|U p(v, \Phi)\|_{l_{2}}^{2}=\|p(v, \Theta)\|_{l_{2}}^{2},
$$

from which condition (13) follows with $\gamma=a / B$.

The converse of Theorem 4 is also true; i.e., any two frames of $\mathscr{H}$ are related by an operator $U$ on $l_{2}$ that satisfies condition (13). The following result, 
together with Theorem 4, gives a complete characterization of all the frames of a given Hilbert space $\mathscr{H}$. It also gives a method for constructing frames with some prescribed properties. As an illustration, in $\S 4.2$ we will use a simple operator $U$ to construct scaling functions and wavelets with some specifiable properties. The converse of the theorem above states:

Theorem 5. If $\left\{\theta_{n}\right\}_{n \in Z}$ and $\left\{\phi_{n}\right\}_{n \in Z}$ are frames for $\mathscr{H}$, then there exists a bounded linear operator $U$ from $l_{2}$ into itself such that

$$
\boldsymbol{\Theta}=U \Phi
$$

Proof. Let $X \subset l_{2}$ denote the range of $p(\cdot, \Phi)$. Because $\left\{\phi_{n}\right\}_{n \in Z}$ is a frame, the operator $p(\cdot, \Phi)$ satisfies (14). The left inequality of (14) implies that $p(\cdot, \Phi)$ is injective and bounded below by the positive constant $A$. Thus, $p(\cdot, \Phi)$ has an inverse $p^{-1}$ from $X$ into $\mathscr{H}$ which is also bounded. From this last assertion, we conclude that $X$ is a closed subspace of $l_{2}$. We define the infinite matrix with entries $u_{i j}$ by

$$
u_{i j}=\left\langle S^{-1} \theta_{i}, \phi_{j}\right\rangle_{\mathscr{C}}
$$

where $S^{-1}$ is the positive operator defined by (2). We define an operator $U$ on $X$ by assigning to every vector $x \in X$ the sequence $(U x)_{i}=\sum_{j} u_{i j} x_{j}$. Because $\left\{\theta_{n}\right\}_{n \in Z}$ is a frame of $\mathscr{H}$, we can use (17) and (2) to obtain

$$
\|U x\|_{l_{2}}^{2}=\sum_{n \in Z}\left|\left\langle\theta_{n}, v\right\rangle_{\mathscr{L}}\right|^{2} \leq b\|v\|_{\mathscr{H}}^{2},
$$

where $v \in \mathscr{H}$ is such that $x=p(v, \Phi)$, and where $b$ is an upper frame bound for $\left\{\theta_{n}\right\}_{n \in Z}$. Using Equations (14) and (22), we conclude that $U$ is a bounded linear mapping from $X \subset l_{2}$ to $l_{2}$ :

$$
\|U x\|_{l_{2}}^{2} \leq \frac{b}{A}\|x\|_{l_{2}}^{2}
$$

Moreover, because of (2) and (21), we have $\boldsymbol{\theta}=U \Phi$ by construction. Finally, since $X$ is closed, the operator $U$ can be extended to all of $l_{2}$ by requiring $U x=0$ for all $x \in X^{\perp}$ (i.e., $X^{\perp}$ is the null space of $U$ ).

\section{EXAMPLES FROM AFFINE FRAMES}

There are many applications of affine frames to signal processing, image processing, and numerical analysis $[4,6-8,11,13,14,16-18]$. In this section, we will illustrate the results of the previous sections by giving examples from affine frames.

4.1. Tight shift-invariant frames and tight frame multiresolutions. Shiftinvariant frame multiresolutions (FMRA) were introduced by J. Benedetto and S. Li [7]. A simple example of Theorem 3 in which a tight FMRA can be obtained by orthogonal projection of a basis is given by the sinc function. Specifically, $\left\{\phi_{n}=\operatorname{sinc}(x-n)\right\}_{n \in Z}$ is an orthogonal basis of the bandlimited function space $B_{1 / 2}$ with bandwidth $W=\left[-\frac{1}{2}, \frac{1}{2}\right]$. The space $B_{1 / 4}$ of bandlimited functions with bandwidth in $W=\left[-\frac{1}{4}, \frac{1}{4}\right]$ is included in $B_{1 / 2}$. By orthogonal projection of $\left\{\phi_{n}\right\}_{n \in Z}$ on $B_{1 / 4}$, we obtain the set $\left\{\theta_{n}=\operatorname{sinc}\left(\frac{x}{2}-\frac{n}{2}\right)\right\}_{n \in Z}$, which, by Theorem 3 , is a tight frame of $B_{1 / 4}$ with a redundancy factor $r=2$. 
This corresponds to oversampling $B_{1 / 4}$ at twice the Nyquist rate. In fact, the sets $\left\{\phi_{n, j}=\operatorname{sinc}\left(2^{-j} x-\frac{n}{2}\right)\right\}_{n, j \in Z}$ are tight frames for the multiresolution of bandlimited functions (cf. [1,2]).

4.2. Construction of scaling functions and wavelets with specifiable properties. A simple example of operator $U$ satisfying the condition of Theorem 4 is obtained as follows: if the Fourier transform $\hat{q}(f)$ of a real sequence $q(k)$ is such that ess $\sup |\hat{q}(f)|<\infty$, then the operator $U_{q} x:=q * x$ (obtained by convolving $q$ with $x$ ) is bounded on $l_{2}[1,3]$, and $U_{q}^{*}=U_{q^{\vee}}$, where $q^{\vee}(k)=q(-k)$. Moreover, if ess $\inf |\hat{q}(f)|>0$, then $U_{q}$ satisfies Theorem 4. Thus, we can use such operators to generate frames (cf. (10)) of a given Hilbert space. However, these types of operators cannot transform a Riesz basis into a redundant frame (or vice versa). To do this, it is necessary to use other types of operators that satisfy Theorem 4.

Using the convolution operators described above, it is possible to construct scaling functions and wavelets with desired properties. In particular, given the orthonormal basis $\left\{\varphi_{o}(x-n)\right\}_{n \in Z}$ of the space $V_{0}$ that belongs to the multiresolution $V_{j}$ [13], we can obtain the interpolating basis $\left\{\varphi_{I}(x-n)\right\}_{n \in Z}$ by using the sequence $q_{I}$ defined by its Fourier transform $\hat{q}_{I}(f)$

$$
\hat{q}_{I}(f)=\left(\sum_{i \in Z} \hat{\varphi}_{o}(f-i)\right)^{-1} .
$$

The type of operators $U_{q}$ described above together with two other types of operations were used to find a general method to construct scaling and wavelet functions with specifiable properties $[2,3]$.

4.3. Nonorthogonal projections. Another example of Theorem 1 is given by nonorthogonal projectors. In particular, we consider the projection $P_{1 \perp 2}$ on the vector space $V_{1}=\left\{\sum_{n \in Z} c(n) \lambda_{1}(x-n)\right\}$ in the direction orthogonal to $V_{2}=\left\{\sum_{n \in Z} c(n) \lambda_{2}(x-n)\right\}$. The spaces $V_{i}$ are well defined, closed subspaces of $L_{2}$ if there exists two positive constants $0<m \leq M$ such that the Fourier transforms $\hat{\lambda}_{i}(f)$ of $\lambda_{i}(x)(i=1,2)$ satisfy [1]

$$
m \leq \sum_{n \in Z}\left|\hat{\lambda}_{i}(f+n)\right|^{2} \leq M \quad \text { a.e. }
$$

The projection $P_{1 \perp 2} g=\sum_{i \in Z} c_{g}(i) \lambda_{1}(x-i)$ of a function $g(x) \in L_{2}$ must satisfy

$$
\left\langle\left(p_{1 \perp 2} g-g\right)(x), \lambda_{2}(x-l)\right\rangle_{L_{2}}=0, \quad \forall l \in Z .
$$

Equation (25) is equivalent to solving the discrete convolution equation for the unknown sequence $c_{g}$ :

$$
c_{g} * a_{12}=b,
$$

where $a_{12}(k)=\left\langle\lambda_{1}(x), \lambda_{2}(x-k)\right\rangle_{L_{2}}$ is the sampled cross-correlation between $\lambda_{1}$ and $\lambda_{2}$, and where $b(k)=\left\langle g(x), \lambda_{2}(x-k)\right\rangle_{L_{2}}$. Equation (26) is the basis of a new technique for sampling signals with nonideal acquisition devices [15].

From Equation (26), an argument similar to the one in [1, Theorem 2] gives the following result: 
Theorem 6. The projector $P_{1 \perp 2}$ is well defined if and only if there exist two positive constants $0<m^{\prime} \leq M^{\prime}$ such that the Fourier transform of the crosscorrelation $\hat{a}_{12}=\sum_{n \in Z} \hat{\lambda}_{1}(f+n) \overline{\hat{\lambda}_{2}(f+n)}$ satisfies

$$
m^{\prime} \leq\left|\sum_{n \in Z} \hat{\lambda}_{1}(f+n) \overline{\hat{\lambda}_{2}(f+n)}\right| \leq M^{\prime},
$$

where $\overline{\hat{\lambda}_{2}(f)}$ is the complex conjugate of $\hat{\lambda}_{2}(f)$.

From (25) and (26), it follows that $P_{1 \perp 2}^{*}=P_{2 \perp 1}$. Thus, $\left\|P_{1 \perp 2}^{*} g\right\|_{L_{2}} \geq\|g\|_{L_{2}}$ for all $g \in V_{1}$. In fact, we have that $\left\|P_{1 \perp 2}^{*} g\right\|_{L_{2}} \geq M^{-1}\|g\|_{L_{2}}$. Thus, by Theorem 2, such projectors can be used to generate frames of $V_{1}$ from frames or bases of $L_{2}$.

\section{ACKNOWLEDGMENTS}

I wish to thank Michael Unser for useful discussions. I also wish to thank Jane Bradley, Barry Bowman, and Maurits Malfait for their editorial help.

\section{REFERENCES}

1. A. Aldroubi and M. Unser, Sampling procedures in function spaces and asymptotic equivalence with Shannon's sampling theory, Numer. Funct. Anal. Optim. 15 (1994), 1-21.

2. - Families of wavelet transforms in connection with Shannon's sampling theory and the Gabor transform, Wavelets-A Tutorial in Theory and Applications, 2 (C. K. Chui, ed.), Academic Press, New York, 1992, pp. 509-528.

3. __ Families of multiresolution and wavelet spaces with optimal properties, Numer. Funct. Anal. Optim. 14 (1993), 417-446.

4. A. Aldroubi, M. Unser, and M. Eden, Discrete spline filters for multiresolutions and wavelets of $l_{2}$, SIAM J. Math. Anal. 25 (1994), 1412-1432.

5. J. Benedetto, Gabor representation and wavelets, Commut. Harmonic Anal. 19 (1989), 9-27.

6. J. J. Benedetto, Irregular sampling and frames, Wavelets-A Tutorial in Theory and Applications, 2 (C. K. Chui, ed.), Academic Press, New York, 1992, pp. 445-507.

7. J. J. Benedetto and S. Li, Multiresolution analysis frames with applications, IEEE-ICASSP 3 (1993), 304-307.

8. I. Daubechies, The wavelet transform, time-frequency localization and signal analysis, IEEE Trans. Inform. Theory 36 (1990), 961-1005.

9. __ Ten lectures on wavelets, CBMS-NSF Regional Conf. Ser. in Appl. Math., SIAM, Philadelpha, PA, 1992.

10. R. J. Duffin and A. C. Schaeffer, A class of nonharmonic Fourier series, Trans. Amer. Math. Soc. 72 (1952), 341-366.

11. C. E. Heil and D. F. Walnut, Continuous and discrete wavelet transforms, SIAM Rev. 31 (1989), 628-666.

12. S. G. Mallat, Multiresolution approximations and wavelet orthogonal bases of $L_{2}(\mathbb{R})$, Trans. Amer. Math. Soc. 315 (1989), 69-87.

13. _ A theory of multiresolution signal decomposition: the wavelet representation, IEEE Trans. Pattern Anal. Machine Intell. PAMI-11 (1989), 674-693.

14. W. Sweldens and R. Piessens, Quadrature formulae and asymptotic error expansions for wavelet approximations of smooth functions, SIAM J. Numer. Anal. 31 (1994), 1240-1264.

15. M. Unser and A. Aldroubi, A general sampling theory for nonideal acquisition devices, preprint.

16. M. Unser, A. Aldroubi, and M. Eden, On the asymptotic convergence of B-spline wavelets to Gabor functions, IEEE Trans. Inform. Theory 38 (1992), 864-872. 
17. _ A family of polynomial spline wavelet transforms, Signal Process. 30 (1993), 141-162.

18. M. Vetterli and C. Herley, Wavelets and filter banks, IEEE Trans. Signal Proc. 40 (1992), 2207-2231.

Beip/NTTT, Bldg. 13, Rm. 3N07A, National Institutes of Health, Bethesda, MaryLAND 20894

Current address: NIH/BeIP, BuILDING 13/3N17, 13 South DR MSC 5766, Bethesda, MARYLAND 20892-5766

E-mail address: ALDROUBI@HELIX.NIH.GOV 